\title{
Grado de conocimiento sobre la propuesta de sustitución del gas de uso doméstico por energía eléctrica entre los habitantes de
} Azogues

\section{Degree of knowledge about the proposal of substitution of the gas of domestic use by electric energy between the inhabitants of Azogues}

\author{
Molina Hugo, Jhon Genaro ${ }^{1 *}$, Palomeque Cantos, René Patricio ${ }^{1}$ y Méndez Vélez, Froilán Segundo ${ }^{1}$ \\ ${ }^{1}$ Universidad Católica de Cuenca, sede Azogues \\ *jgmolinah@ucacue.edu.ec
}

DOI: https://doi.org/10.26871/killkana_social.v2i1.75

\begin{abstract}
Resumen
El presente estudio, tuvo por finalidad identificar el grado de conocimiento de los habitantes de la ciudad de Azogues, con respecto a la propuesta de sustitución del gas de uso doméstico por energía eléctrica por parte del Gobierno de Rafael Correa, así como el nivel de aceptación de la población sobre esta nueva política económica. El presente estudio alcanzó un nivel descriptivo, aplicándose una encuesta de preguntas cerradas a una muestra poblacional de 191 hombres y 192 mujeres de la ciudad de Azogues, tanto del sector urbano como del rural. Se obtiene como resultado que el 98,99\% no está de acuerdo con que se elimine el subsidio al gas, concluyéndose que el Gobierno no ha colectivizado de forma adecuada la información que permita a la ciudadanía tomar una decisión o asumir una posición frente al cambio. En tal sentido, sería indispensable profundizar los programas de difusión, los cuales, si bien existen, no son suficientes. De ahí la necesidad de implementar el debate popular, y motivar la razón del porqué de este cambio.
\end{abstract}

Palabras clave: Economía, ahorro, consumo, energía, gasto público, política económica, conocimiento, cocinas.

\begin{abstract}
The present study was aimed to identify the level of knowledge of the city of Azogues' inhabitants regarding the proposal to replace domestic gas use by electric energy as suggested by the Government of Rafael Correa; as well as the level of population's acceptance of this new economic policy. The present study reached a descriptive level through the application of a closed questionnaire to a population sample of 191 men and 192 women from the city of Azogues both, from the urban and rural areas. The results obtained show that 98, 99\% of the population does not agree with the elimination of the gas subsidy, concluding that the Government has not properly collectivized the information that allows citizens to make a decision or take a position on the change. In this sense, it would be essential to deepen the information spread programs, which, although they exist, are not enough; Hence the need to implement the popular debate, to motivate the reason for this change.
\end{abstract}

Key words: Economy, savings, consumption, energy, public expenditure, economic policy, knowledge, stoves.

\section{Introducción}

Durante los últimos años el gobierno ecuatoriano ha implementado varias medidas económicas, una de las principales, el programa de sustitución de cocinas a gas licuado de petróleo $(\mathrm{GLP})^{1}$ por cocinas de inducción. A través de este programa se busca eliminar el subsidio al gas, el que representa 700 millones de dólares americanos al
año(Salvador, 2015). Según lo planeado por el gobierno, este programa entraría en vigor desde el año 2016, eliminando por completo el subsidio al gas licuado de petróleo y dando paso al subsidio porcentual de energía eléctrica doméstica, que se extenderá hasta el año 2018(El Comercio, 2013). Esto trae consigo un sinnúmero de incógnitas, generando gran especulación entre la población.

${ }^{1}$ Consiste en la mezcla de gases licuados presentes en el gas natural o, a su vez, disueltos en el petróleo (nota del autor). 
Pese a la importancia del tema considero que hay poco conocimiento de la ciudadanía con respecto a la medida, así como poca aceptación de la política a implementarse.

El Ecuador se caracteriza por ser exportador de bienes primarios de bajo valor agregado, a la vez que importador de bienes industrializados(Acosta, 2012). La producción nacional representa el $90 \%$ de la oferta energética total, la que está concentrada en un $96 \%$ en petróleo crudo y gas natural, mientras que las energías renovables (hidroelectricidad y biomasa) alcanzan un $4 \%$ de la producción nacional. Las importaciones constituyen el $10 \%$ restante de la oferta, de las cuales, el $90 \%$ corresponde a derivados de petróleo (GLP, diésel, nafta de alto octano y otros)(Centrosur, 2015).

Con respecto a la demanda energética, el $64 \%$ corresponde a las exportaciones, el $28 \%$ a la demanda doméstica y el $8 \%$ a pérdidas por transformación. De las exportaciones, el $90 \%$ corresponde a petróleo crudo, el $9 \%$ a restantes de derivados de bajo valor agregado y el $1 \%$ a aceites de origen vegetal. Por su parte, la demanda doméstica se compone de derivados de petróleo $(79 \%)$, electricidad $(13 \%)$, biomasa (5\%), y productos como carburantes, entre otros ( $2 \%)$; además, se concentra en los sectores transporte $(52 \%)$, industria $(21 \%)$, residencial $(19 \%)$, comercial y servicios (4\%), y otros (4\%)(Ministerio de Electricidad y Energía Renovable, 2016).

El cambio de la matriz energética en el Ecuador se fundamenta en varios criterios: en la ejecución inmediata de los proyectos hidroeléctricos del Plan Maestro de Electrificación, en el impulso a los proyectos de utilización de otras energías renovables, en la reducción de las importaciones de derivados de petróleo, en el empleo del crudo de petróleo como insumo en la nueva Refinería del Pacífico, en el trabajo sobre el sector del transporte, industrial y residencial, y, finalmente, en la generación de conciencia entre la población de un ahorro energético acorde a un consumo sustentable (Secretaría Nacional de Planificación y Desarrollo, 2013).

Por su parte, la sustitución de las cocinas a gas por cocinas de inducción encontraría su justificación en que mientras el Estado importa cada cilindro de 15 kilos en USD 22,27, el precio oficial en el país es USD 1,60, por lo que el presupuesto destinado al subsidio al gas bordea los USD 700 millones al año. A su vez, cada hogar ecuatoriano consume 1,16 cilindros de gas al mes en promedio para la cocción de alimentos, lo que transformado a energía eléctrica equivale a 209 kilovatios - hora (kW-h) al mes. Por otro lado, únicamente el $50 \%$ de la energía producida con gas se aprovecha; el $50 \%$ restante pierde como calor. Así mismo, el $92 \%$ del GLP utilizado en Ecuador se consume en el sector residencial, no obstante, el país importa cerca del $80 \%$ de la demanda de dicho combustible, debido a la insuficiente producción nacional(Ministerio de Electricidad y Energía Renovable, 2015).

Otra razón importante es el contrabando de cilindros de gas existente en las fronteras de Colombia y Perú, lo que se debe a que en ambos países el mismo cilindro cuesta entre \$15 y \$20 (Andrade, 2011). A su vez, los pobres reciben un $22 \%$ del porcentaje del subsidio, frente al $58 \%$ recibido por las personas de ingresos medio alto y alto (Plan V, 2015). Finalmente, como el gas procede del petróleo requiere de una fuente no renovable.

Frente a las condiciones señaladas, el Gobierno ecuatoriano se planteó como solución la financiación de cocinas hasta un plazo de tres años, con cuotas mensuales que varían entre los USD 6,5 y USD 18, dependiendo del modelo escogido, y que podrán ser canceladas junto con el pago de las planillas (Ministerio de Electricidad y Energía Renovable, 2015). Al mismo tiempo, el Ministerio de Electricidad y Energía Renovable, por medio de las empresas eléctricas, reforzará las redes e instalará, sin costo, acometidas y medidores de 220 voltios (Empresa Eléctrica Quito, 2014). Para esto se calcula una inversión de USD 485 millones (El Telégrafo, 2015). A su vez, entrarán en operación ocho nuevos proyectos hidroeléctricos que garantizarán la provisión de energía limpia y renovable (El Telégrafo, 2015).

Por otro lado, una cocina de inducción solo requiere $120 \mathrm{~kW}-\mathrm{h}$ al mes para cocinar lo mismo que 1,16 cilindros de gas. De estos $120 \mathrm{~kW}$-h, los primeros $80 \mathrm{KW}$ serán gratuitos hasta enero del 2018. El ciudadano terminará pagando aproximadamente USD 4 adicionales a su planilla habitual, aunque al mismo tiempo dejará de comprar gas (Ministerio de Electricidad y Energía Renovable, 2015). A su vez, si opta por pasarse de calefón a ducha eléctrica, el Gobierno le subsidiará al ciudadano $20 \mathrm{KW}$ mensuales (Ministerio de Electricidad y Energía Renovable, 2015).

Como ventajas de las cocinas de inducción se señalan: su mayor seguridad, eliminándose los riesgos de fugas, intoxicaciones y explosiones; su fácil limpieza, ya que los alimentos derramados no se pegan; su mayor eficiencia, pues aprovecha el $85 \%$ de la energía (Ministerio de Electricidad y Energía Renovable, 2015); su rapidez para cocinar así como su fácil uso, en razón de que cuenta con un teclado que permite un mejor control de la temperatura; su comodidad e independencia, debido a que no se tiene que cargar el cilindro ni se depende del distribuidor de gas; $\mathrm{y}$, finalmente, sus propiedades estéticas que le otorgan a la cocina un aspecto moderno y agradable (Ministerio de Electricidad y Energía Renovable, 2015).

Al respecto de la medida, algunos funcionarios del Gobierno ecuatoriano se han pronunciado. Esteban Albornoz, Exministro de Electricidad y Energía Renovable, señaló que esta medida no implicaría ningún impacto social. Argumenta que, si ahora cuesta, por ejemplo, dos dólares cocinar con gas, con electricidad va a costar exactamente igual o algo menos (El Mercurio, 2013). Por su parte, Andrés Arauz, Exministro Coordinador de Conocimiento y Talento Humano, destacó que la medida generará empleo especializado, pues se requerirá una gran cantidad de ingenieros eléctricos para hacer los cambios requeridos (Radio Ciudadana, 2015). Por su parte, Jorge Glas, destaca el hecho que la propuesta reducirá 11 toneladas de dióxido 
de carbono (CO2) al año o su equivalente al $10 \%$ de la contaminación emitida por todos los transportes (El Universo, 2014), explicó.

A continuación, se hace un repaso a estudios que respaldan la medida: (Tama, 2014) señala que es necesario direccionar de manera adecuada las políticas para la aplicación del actual programa de sustitución tecnológica, considerando además que los ahorros para el Estado conllevarán a "ingentes inversiones en el sector eléctrico para suplir el incremento de la demanda de potencia y energía" (pág. 14)

(Serrano y Rojas, 2013), por su parte, señalan que es urgente repotenciar el sistema de generación, transmisión y distribución de energía eléctrica, a través de la elevación de voltaje con el fin de mantener los mismos calibres de conductores actuales. Así mismo, recomiendan reemplazar los sistemas de transformación de tensión e incorporar tarifas diferenciadas de energía por horario, con el objeto de modular la curva de carga.

(Riofrío, Carrión, Orozco, Vaca, y Martínez, 2014) demuestran que el empleo de la tecnología de cocción por inducción, en el caso de un equipo con potencia limitada necesita menor energía eléctrica en comparación con la cocina a base de GLP; sin embargo, el tiempo requerido para la cocción es mayor. Cuando se utiliza el mismo equipo, liberándolo de su limitación de potencia se alcanzan valores mínimos con respecto a la energía consumida y tiempo de ensayo, lo que convierte a este equipo en la mejor opción en términos energéticos.

Por su parte, (Tello, 2015) evidenció que la cocina de inducción posee ventajas frente a la de gas, ahorrando hasta 36 kwh al mes, no entraña peligro alguno (Tama, 2014; Serrano y Rojas, 2013), pues no emplea combustión para la cocción de alimentos, no implica peligros de muerte por inhalación de gases venenosos y es más rápida en la cocción. Pero también tiene sus desventajas: no es portable, puesto que requiere de una instalación eléctrica hecha por personal especializado, al tiempo que necesita de cacerolas con bases ferromagnéticas, lo que eleva su costo de implementación.

Por su lado, (Plaza, Escobar, y Aguirre, 2015) apuntan como desventaja de la cocina de inducción el hecho que no existe un adecuado asesoramiento del empleo eficiente de la cocina por parte de las empresas comercializadoras al momento de realizar la compra e instalación. Otro punto interesante señalado por los autores, es que la incorporación de las cocinas de inducción tiene un mayor impacto en los picos de la curva de carga diaria del Ecuador en razón de que los horarios de preparación de los alimentos coinciden con la misma, con lo cual se perjudica al factor de carga.

Finalmente, (Calderón, Alcívar, y Chapin, 2016) realizan un listado de lo que podría ocurrir en el Ecuador durante el proceso de transición de la cocina a gas a la de inducción: la eliminación de todas las cocinas a gas existentes en el país, la pérdida de empleos y la adquisición de nuevos utensilios de cocina.

\section{Método}

El presente estudio alcanza un nivel descriptivo, en razón que se limitará a describir el fenómeno estudiado, esto es, el nivel de aceptación y conocimiento de los hogares de la ciudad de Azogues con respecto a la transición del sistema de cocción de gas a sistema de cocción por inducción, sin establecer las causales que podrían estar detrás de esta situación, lo que se dejaría para una futura investigación.

A su vez, se aplicará una encuesta de preguntas cerradas sobre aspectos varios relacionados al nuevo sistema de cocción por inducción y al nuevo sistema por implementarse en los hogares ecuatorianos.

En base a datos del (INEC, 2010) se estableció que el universo poblacional de la ciudad de Azogues es de 70.064 habitantes. A partir de dicho universo se calculó la muestra, la que con un porcentaje de error del $5 \%$, un nivel de confianza del $5 \%$, con una distribución de las respuestas del $50 \%$, se constituye en 383 personas. Para asegurar un equilibrio en las respuestas se procedió a dividir la muestra en 191 hombres y 192 mujeres, al tiempo que se visitó las distintas parroquias del sector urbano como Aurelio Bayas, Azogues, Borrero, San Francisco; así como las del sector rural: Cojitambo, Guapán, Javier Loyola, Luis Cordero, Pindilig, Rivera, San Miguel y Taday.

\section{Análisis de resultados}

El presente estudio busca identificar el grado de conocimiento de los habitantes de Azogues con respecto a la propuesta de sustitución del gas de uso doméstico por energía eléctrica por parte del Gobierno de Rafael Correa, así como el nivel de aceptación de la población sobre esta nueva política económica

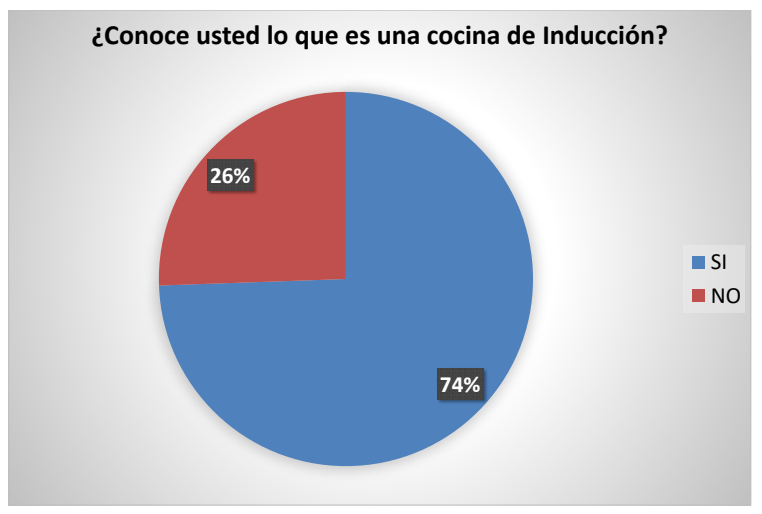

Figura 1. Conocimiento sobre las cocinas de inducción

Fuente: Encuesta a la población de Azogues

Pese a la campaña de socialización de la medida implementada por el Gobierno, existe todavía un porcentaje significativo de habitantes que desconocen las propiedades de las cocinas de inducción. A su vez, aunque el $74 \%$ 
señala conocer qué es una cocina de inducción, se pudo constatar que la información es incompleta, esto es, no se conoce las ventajas que tienen las cocinas de inducción.

Únicamente el $6 \%$ respondió que es obligatorio el cambio de las cocinas de gas a cocinas de inducción. El resto de los encuestados aciertan al referir que no existe obligatoriedad por parte de la población de emplear las cocinas de inducción. (Figura 2)

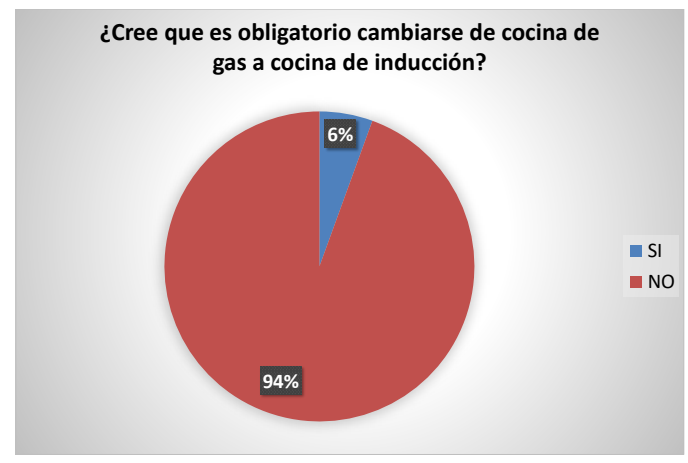

Figura 2. Conocimiento sobre la obligatoriedad de cambiarse a cocina de inducción

Fuente: Encuesta a la población de Azogues

Aunque la mayoría de los encuestados señala conocer el precio aproximado de una cocina de inducción, existe un porcentaje significativo $(43 \%)$ que desconoce dicho valor, lo que permite suponer que no han establecido un presupuesto que les permita enfrentar los cambios que se avecinan.

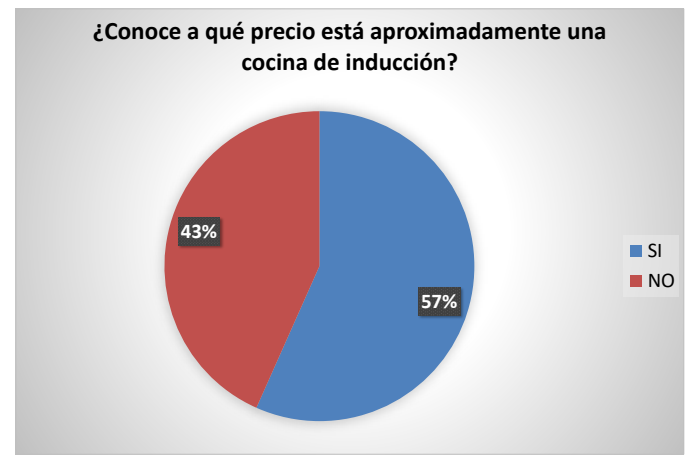

Figura 3. Conocimiento sobre el precio aproximado de una cocina de inducción

Fuente: Encuesta a la población de Azogues

A su vez, el $57 \%$ de la población conoce el precio que conlleva acceder a estos nuevos electrodomésticos con sus respectivos accesorios; sin embargo, no acepta la idea de la adquisición de estos electrodomésticos, negándose a desechar las cocinas y las ollas actualmente utilizadas. Al respecto, el Gobierno ha recomendado no desechar las cocinas a gas en caso de darse algún imprevisto (Expreso, 2015).

Como medida estratégica el gobierno ha establecido planes de crédito para la obtención de estos electrodomésticos junto con sus accesorios (ollas) (El Telégrafo, 2017). Es claro observar que el Gobierno está empleando diversas estrategias para incentivar a la población al cambio en el sistema utilizado.

El $59 \%$ desconoce el tipo de mantenimiento que se le debe dar a las cocinas de inducción. (Figura 4)

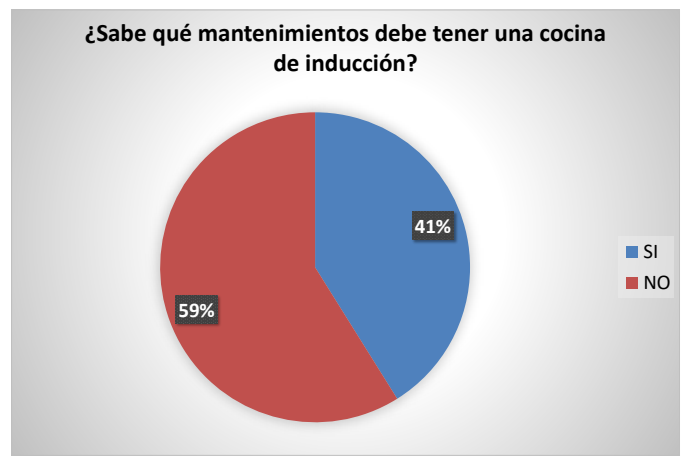

Figura 4. Conocimiento sobre los mantenimientos que debe tener una cocina de inducción

Fuente: Encuesta a la población de Azogues

Al respecto, hay que señalar que las cocinas de inducción solo requieren de una limpieza básica superficial sobre el vidrio o la vitrocerámica con un paño húmedo. Al no tener piezas móviles, no requiere de mayor mantenimiento; a más de esto, la vida útil de una cocina de inducción, antes de presentar algún desperfecto, es de un periodo de 15 años, es decir, el mantenimiento de uno de estos nuevos electrodomésticos no sería un impedimento (El Comercio, 2014).

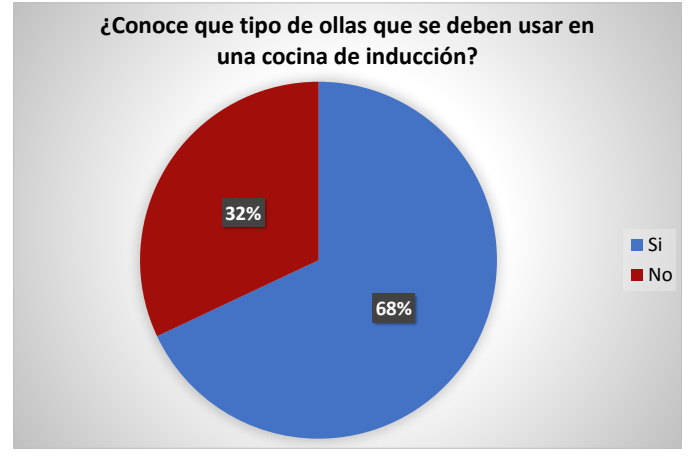

Figura 5. Conocimiento sobre tipos de ollas

Fuente: Encuesta a la población de Azogues

El $68 \%$ responde que sí conocen el tipo de ollas que se deben usar en las cocinas de inducción, frente a un $32 \%$ 
que no lo sabe (Figura 5). Es importante señalar que los accesorios con los cuales se manejan las cocinas de inducción (en este caso, las ollas) conllevan un gasto adicional que se sumaría al valor total de la adquisición de estos nuevos artefactos. Desconocer el tipo de olla requerida implica no estar al tanto del costo que conlleva dicho accesorio.

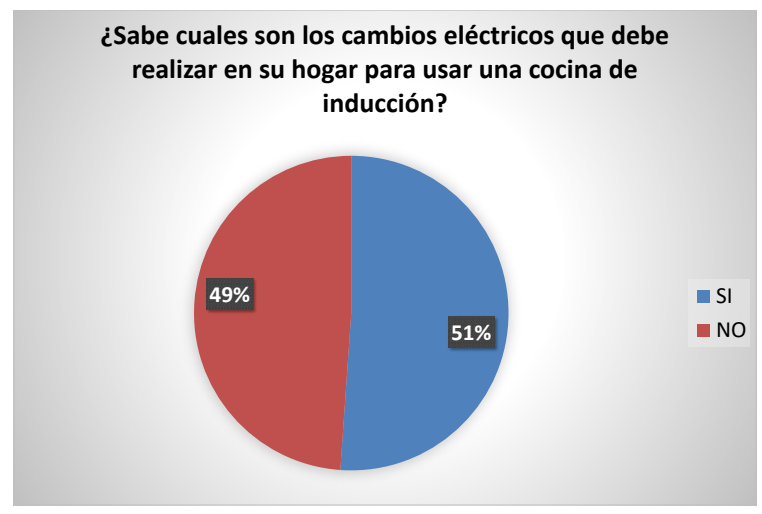

Figura 6. Conocimiento sobre el precio aproximado de una cocina de inducción

Fuente: Encuesta a la población de Azogues

El correcto funcionamiento de las cocinas de inducción conlleva a un cambio en la adecuación de las conexiones. Para instalar los conductores de electricidad de 220 voltios se deben emplear ciertos materiales. Esto es desconocido por la mitad de los encuestados, lo que resulta preocupante, pues evidencia una falta de preparación para enfrentar los cambios físicos que deben realizarse en los hogares.

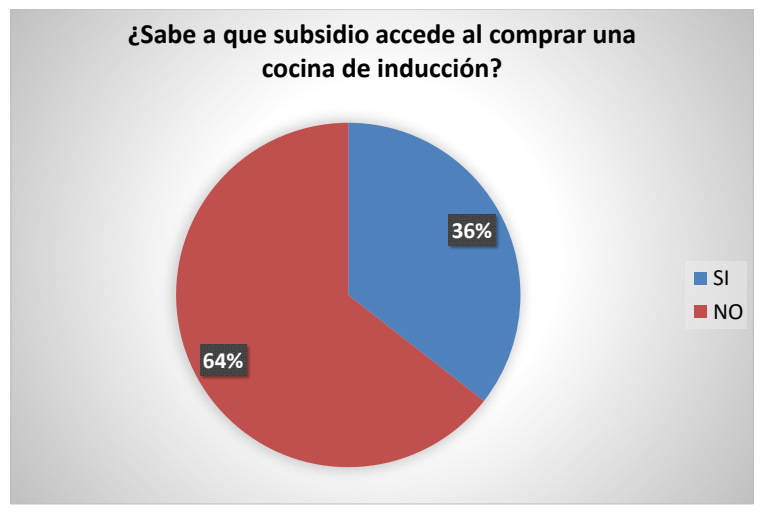

Figura 7. Conocimiento sobre el subsidio al que se accede con la compra de una cocina de inducción

Fuente: Encuesta a la población de Azogues

El Gobierno subsidiará $80 \mathrm{KW}$ para el uso de las cocinas de inducción y $20 \mathrm{KWh}$ para el uso de duchas eléctricas. Según los resultados, el $36 \%$ de la población está al tanto de esta información, frente a un $64 \%$ que la desconoce. Se evidencia que existe una falta de socialización en una parte significativa de la población, la que desconoce las estrategias utilizadas por el gobierno para la implementación de este sistema. El Gobierno ha propuesto subsidiar los primeros $80 \mathrm{KW}$ hasta enero del 2018, por lo que el ciudadano terminaría pagando cerca de USD 4 adicionales en su planilla, aunque, por otro lado, dejará de comprar gas. El Gobierno también subsidiará $20 \mathrm{KW}$ para el uso de duchas eléctricas, en caso de que el ciudadano optase por el cambio de calefones (figura 7).

Solamente el $36 \%$ de la población conoce sobre este subsidio, el resto desconoce esta información que resulta relevante en la toma de una decisión sobre esta medida económica de gran impacto social, ello provoca la aparición de especulaciones y la falta de formación de un criterio rígido sobre las decisiones tomadas.

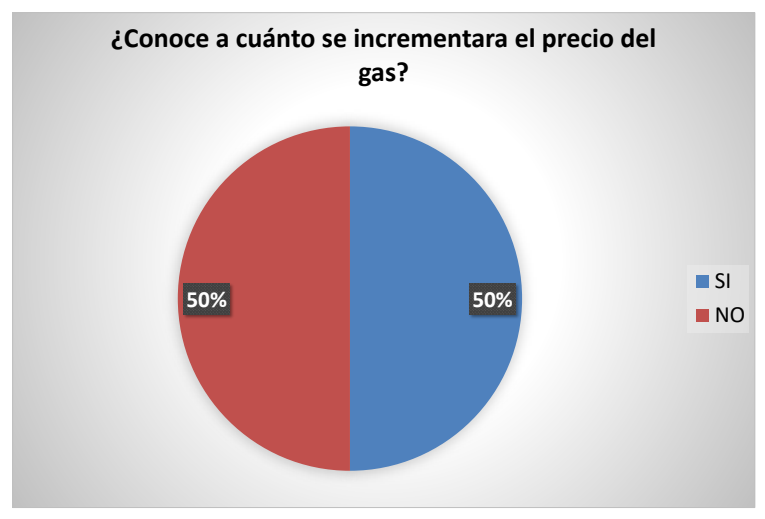

Figura 8. Conocimiento sobre la cantidad en que se incrementará el precio del taque de gas

Fuente: Encuesta a la población de Azogues

La mitad de la población afirma conocer la cantidad en que se incrementará el precio del cilindro de gas licuado de petróleo. Al respecto, cabe mencionar que el precio de los cilindros de gas aumentaría a \$22,27 por unidad (Idrovo, 2014). Con base en estos datos, puede evidenciarse que aquellos que indican que el precio del cilindro de gas llegaría a los \$20, \$18 o \$25, no disponen de información correcta, aunque muy cercana a la realidad (figura 8).

Casi la totalidad de los encuestados $(98,99 \%)$ está en desacuerdo con la eliminación del subsidio al gas (figura9). Se puede deducir que, aun con el grado de conocimiento que posee una gran parte de la población sobre los beneficios de la implementación de las cocinas de inducción, la mayoría no está de acuerdo con dicha política económica. El acatamiento sería una obligación necesaria resultante de los costos que implicaría seguir utilizando los métodos de cocción actuales. El Gobierno establece que el monto de dinero que actualmente invierte en el subsidio al gas, podría invertirse para crecer y crear un beneficio social, viéndolo como un presupuesto de inversión; enfocado al desarrollo económico del País. 


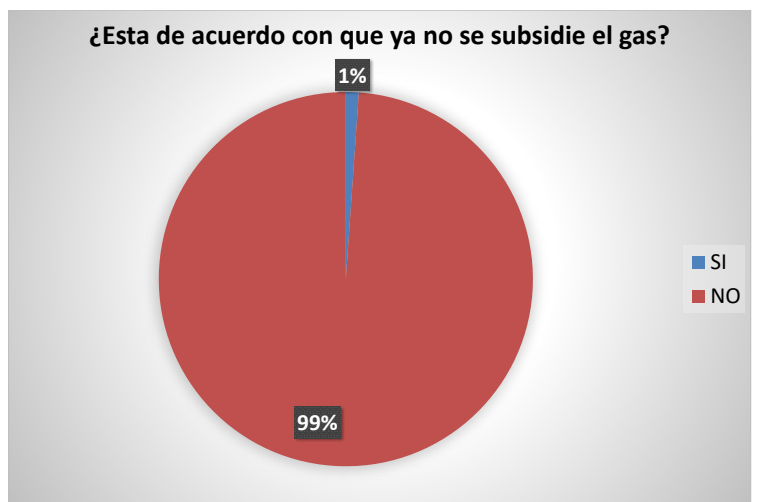

Figura 9. Conformidad con quitar el subsidio al gas Fuente: Encuesta a la población de Azogues

\section{Conclusiones}

El Gobierno ecuatoriano se ha visto forzado a tomar ciertas decisiones en razón de que la situación económica actual cursa por una serie de problemas. Medidas como la analizada en el presente estudio, ayudarían a resolver parte de estos problemas económicos. A través de la sustitución de la cocina de gas por la cocina de inducción se busca modificar un subsidio secular por otro más barato y manejable. Con ello se procura rescatar los 700 millones de dólares que se destinan a una subvención permanente y creciente del gas doméstico, ofreciendo energía alternativa limpia y segura. No obstante, todo ello implica cambios económicos y la modificación de ciertas costumbres sociales.

La negativa social entre los hogares de Azogues respecto al cambio, permite suponer que la población entendería la sustitución como un nuevo costo a su economía, algo que no soluciona los problemas ya existentes, sino que los complica. De ahí, podría deducirse que los habitantes de la ciudad de Azogues prefieren al gas tradicional, al entenderlo como un mecanismo habitual, aceptado y probado para la cocina y el baño.

A lo anterior habría que sumar el hecho de que el Gobierno ecuatoriano no ha cumplido con sus propios proyectos, como es el caso de las hidroeléctricas, las que, según el Estado, debían ya encontrarse en funcionamiento, cuando la realidad es otra.

A más de esto, existe el problema de que el Gobierno no ha colectivizado la información necesaria que permita a la ciudadanía tomar una decisión o asumir una posición frente al cambio. En tal sentido, sería indispensable profundizar los programas de difusión, los cuales, si bien existen, no son suficientes; de ahí la necesidad de implementar el debate popular, el motivar la razón del porqué de este cambio.

Como se ha observado, la población investigada no acepta lo formulado, lo cual limita este proceso y lo vuelve todavía impopular.
Aunque se considera que el Programa de eficiencia energética por sustitución resolverá el problema del subsidio, al tiempo que dotará de energía limpia y segura a los hogares, tal apreciación no es generalizada, no tanto por la esencia misma de la propuesta, sino por la falta de un diálogo con la ciudadanía y una capacitación general, acciones que permitirían romper con la idea de que el costo adicional por instalaciones, nuevas ollas, medidor de energía eléctrica, calefón, etc., representa un gasto adicional (de consumo o inversión), sino que es parte de un mismo proyecto.

\section{Referencias Bibliográficas}

Acosta, A. (2012, 31 de julio). Extractivismo y neoxtractivismo: dos caras de la misma maldición. Descargado 1 de Junio de 2016, de http: //www.cronicon.net/paginas/ Documentos/paq2/No.23.pdf

Andrade, S. (2011, enero). El precio social del gas licuado de petróleo en el Ecuador: Crisis de gobernanza. Descargado 4 de Junio de 2016, de http:// 67.192.84.248:8080/bitstream/10469/ 3252/1/TFLACSO-2011SIAH.pdf

Calderón, J., Alcívar, C., y Chapin, M. (2016). El impacto que tendría el cambio de cocinas a gas por inducción en la sociedad ecuatoriana. Descargado 9 de Junio de 2016, de http:// www. eumed. net/cursecon/ecolat/ec/ 2014 /cocinas.zip

Centrosur. (2015). La nueva matriz: Cambio de la matriz energética. Descargado 2 de Junio de 2016, de http://www. centrosur.com.ec/ ? $q=$ node $/ 3$

El Comercio. (2013, 7 de agosto). Fin del subsidio al gas en el 2013. Descargado de http://www .elcomercio.com/opinion/editorial/ del-subsidio-al-gas-2016.html

El Comercio. (2014, 1 de julio). Las 10 preguntas más frecuentes sobre las cocinas de inducción. Descargado 3 de Junio de 2017, de http://www.elcomercio.com/ actualidad/ecuador-negocios-cocinas -induccion-preguntas.html

El Mercurio. (2013, 19 de Agosto). Tendrá el mismo precio cocinar con las cocinas a electricidad. Descargado 16 de Mayo de 2017, de http: / / www.elmercurio.com.ec/393789 -tendra-el-mismo-precio-cocinar-con -las-cocinas-a-electricidad/

El Telégrafo. (2015, 30 de Agosto). 5 obras impulsan cocción eficiente en la zona. Descargado de

http://www.eltelegrafo.com.ec/ noticias/regional-centro/1/ 5-obras-impulsan-coccion-eficiente -en-la-zona 
El Telégrafo. (2017, 19 de Enero). El Biess facilita compra de cocinas. Descargado 3 de Junio de 2017, de http://www.eltelegrafo.com.ec/ noticias/economia/8/las-cocinas-de -induccion-se-podran-adquirir-con -credito-quirografario

El Universo. (2014, 25 de Mayo). Jorge Glas cita cambios hacia nueva matriz productiva. Descargado 2 de Junio de 2017, de http://www.eluniverso .com/noticias/2014/05/25/nota/ $3006411 /$ glas-cita-cambios-hacia -nueva-matriz-productiva

Empresa Eléctrica Quito. (2014, 1 de Septiembre). Se ejecuta plan piloto para instalar acometidas y medidores de 220 V en Sucumbíos. Descargado 4 de Junio de 2016, de http: / / www . eeq. com. ec: $8080 /$ nosotros/comunicamos/noticias/ - /asset_publisher/PDd0RO71Su5d/ content/se-ejecuta-plan-piloto-para -instalar-acometidas-y-medidores -de-220-v-en-sucumbios; jsessionid= 72ECC3B20E1B518F45FEA86C432C7F65

Expreso. (2015, 4 de Enero). Gobierno intensifica la campaña para alentar la compra de cocinas de inducción. Descargado 3 de Junio de 2017 , de http://www.expreso.ec/economia/ gobierno-intensifica-la-campana -para-alentar-EXGR_7426064

Idrovo, P. (2014). Introducción de Cocinas Eléctricas de Inducción Magnética en el Ecuador. Quito: Quito.

INEC. (2010). Resultados del Censo 2010. Descargado 19 de Junio de 2016, de http://www.ecuadorencifras.gob.ec/ resultados/

Ministerio de Electricidad y Energía Renovable. (2015). Programa de eficiencia energética para cocción por inducción y calentamiento de agua con electricidad en sustitución del gas licuado de petróleo (GLP) en el sector residencia - 1 . Descargado 3 de Junio de 2016, de http:// www.energia.gob.ec/programa-de -eficiencia-energetica-para-coccion -por-induccion-y-calentamiento -de-agua-con-electricidad-en -sustitucion-del-gas-licuado-de -petroleo-glp-en-el-sector-reside/

Ministerio de Electricidad y Energía Renovable. (2016). Proyectos de generación eléctrica. Descargado de http://www.energia.gob.ec/ proyectos-emblematicos-2/

Plan V. (2015, 19 de Octubre). Los subisidios: ¿amputación con anestesia?

Plaza, R., Escobar, E., y Aguirre, D. (2015). Análisis de eficiencia energética en la implementación de cocinas de inducción en el sistema eléctrico ecuatoriano. Descargado 9 de Junio de 2016, de http: / /www.dspace.espol.edu.ec/ bitstream/123456789/31088/1/

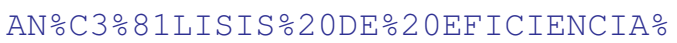
20ENERGC $3 \div 89$ TICA $20 \mathrm{EN} \div 20 \mathrm{LA} \%$ 20 IMP LEMENTACIC $3 \% 93 \mathrm{~N} \div 20 \mathrm{DE} \%$ 20 COCINAS2ODE 20 INDUCCIC $3 \%$ $93 \mathrm{~N} \div 20 \mathrm{EN} \div 20 \mathrm{EL} \div 20 \mathrm{SISTEMA} \div 20 \mathrm{EL} \div \mathrm{C} 3 \%$ 89CTRICO 20 ECUATORIANO.pdf

Radio Ciudadana. (2015, 7 de Noviembre). Uso de cocinas de inducción es uno de los cambios más importantes. Descargado 1 de Junio de 2017, de http://www.laciudadana .gob.ec/index.php/especiales/ informe-a-la-nacion-2015/item/ 5008-uso-de-cocinas-de-induccion-es -uno-de-los-cambios-mas-importantes -de-la-historia-nacional.html

Riofrío, A., Carrión, D., Orozco, M., Vaca, D., y Martínez, J. (2014). Análisis del consumo energético en procesos de cocción eficiente para el sector residencial. Memorias del Congreso latinoamericano de ingeniería mecánica COLIM, VIII, 268-273. Descargado 7 de Junio de 2016, de https://www .researchgate.net/profile/Javier _Martinez26/publication/273438965 _Anlisis_del_consumo_energtico _en_procesos_de_coccin_eficiente _para_el_sector_residencial/links/ 55c0fc1d08aec0e5f44901ec.pdf

Salvador, P. (2015). Investigación de las modalidades de importación de gas licuado de petróleo y su impacto en el presupuesto general del Estado en el periodo 2011-2014. Descargado 1 de Julio de 2016, de http://repositorio.uisek.edu.ec/ jspui/bitstream/123456789/1686/ 1 / TESIS2 OCOMP LETA2 OXAVIER응 20SALVADOR20FINAL . pdf

Secretaría Nacional de Planificación y Desarrollo. (2013). Plan Nacional del Buen Vivir 20132017. Descargado 15 de Enero de 2016, de http://www.buenvivir.gob.ec/ documents/10157/26effa35-aaa8-4aec -a11c-be69abd6e40a

Serrano, J., y Rojas, J. (2013). Impacto de la implementación masiva de la cocina de inducción en el sistema eléctrico ecuatoriano. Descargado 6 de Junio de 2016, de http:// www. iner.gob.ec/wp-content/uploads / downloads/2015/04/ISEREE_Impacto-de -la-implementaciㄷ $3 \% B 3 n-m a s i v a-d e$ -la-cocina-de-inducciC $3 \% \mathrm{~B} 3 \mathrm{n} \cdot \mathrm{pdf}$

Tama, A. (2014). Cocina de inducción versus cocina a gas. Descargado 5 de Junio de 2016, de http://www.dspace.espol.edu.ec/ xmlui/bitstream/handle/123456789/ 
25742 / Cocina 20 de 20 Inducci $\%$ C $3 \% B 3 n \div$ 20 versus $\% 20$ Cocina $20 a \div 20$ Gas $\% 20$ (GLP) -pdf? sequence=1

Tello, D. (2015). Análisis técnico del comportamiento del consumo y la demanda eléctrica en viviendas típicas de la ciudad de Cuenca, debido a la incorporación de la cocina de inducción y sistemas eléctricos para ACS. Descargado 8 de Junio de 2016, de http: / / www. dspace.ups.edu.ec/ handle/123456789/7522

Recibido: 16 de noviembre de 2017

Aceptado: 24 de enero de 2018 